\title{
Is COVID in Neonates Really Mild?
}

\author{
Anilkumar M. Khamkar ${ }^{1} \cdot$ Abhay Mahindre $^{1}$ - P. D. Pote ${ }^{1} \cdot$ Pradeep Suryawanshi $^{1} \cdot$ Georgeena Elsa Jose $^{1}$ (i)
}

Received: 22 May 2021 / Accepted: 15 June 2021 / Published online: 10 August 2021

(c) Dr. K C Chaudhuri Foundation 2021

To the Editor: Nevertheless SARS-CoV-2 affects all age groups, for reasons unidentified at this time, neonates seem to be at a lower risk of severe infection. A systematic review of globally published literature flaunts only 44 reported cases of neonatal SARS-CoV-2 infection [1]. We describe 5 neonates who were hospitalized for COVID-19 in March 2021.

Four neonates were reverse transcriptase polymerase chain reaction (RT-PCR) positive and 1 was rapid antigen test positive for SARS-CoV-2, from their nasopharyngeal samples. Two out of 5, had severe COVID-19 disease.

One among them was the first case reported of neonatal SARS-CoV-2 encephalitis, a 4-d-old baby with convulsions, cerebrospinal fluid pleiocytosis and acute splenial lesions of corpus callosum in the magnetic resonance imaging of brain. We reached this diagnosis after eliminating bacterial sepsis/meningitis, metabolic causes, and other neurotropic viral etiologies. Lindan et al. identified similar lesions in neuroimaging in children with multisystem inflammatory syndrome [2], which was observed for the first time in a neonate with active COVID.

The second case was a 13-d-old baby with severe respiratory distress with hypoxia, myocardial dysfunction, and high-resolution computed tomography chest findings suggestive of COVID-19 pneumonia requiring respiratory and inotropic support. The third neonate was 16 -d -old, who had moderate COVID-19 disease with cough, tachypnea, and similar chest imaging findings.

Other two had mild COVID-19 disease, but required hospitalization, as they had intermittent fever for more than 5 d. All the neonates survived. However, neonates should be placed at a higher risk than children due to the increasing severity of manifestations as described by Dong et al. [3].

Based on these cases, it is imperative to note that more neonates are symptomatic, requiring hospitalization. Secondly, in the second wave of COVID-19 infection in India, we observe a paradigm shift of severity from mild cases to SARS-CoV-2 pneumonia and encephalitis yet with a good prognosis.

\section{Declarations}

Informed Consent Written informed consent is taken from all the 5 parents of the neonates.

Conflict of Interest None.

\section{References}

1. Trevisanuto D, Cavallin F, Cavicchiolo ME, Borellini M, Calgaro S, Baraldi E. Coronavirus infection in neonates: a systematic review. Arch Dis Child Fetal Neonatal Ed. 2021;106:F330-5.

2. Lindan CE, Mankad K, Ram D, et al.; ASPNR PECOBIG Collaborator Group. Neuroimaging manifestations in children with SARS-CoV-2 infection: a multinational, multicentre collaborative study. Lancet Child Adolesc Health.2021;5:167-77.

3. Dong Y, Mo X, Hu Y, et al. Epidemiology of COVID-19 among Children in China. Pediatrics.2020;145:e20200702.

Publisher's Note Springer Nature remains neutral with regard to jurisdictional claims in published maps and institutional affiliations.
Georgeena Elsa Jose

egeorgeena@gmail.com

1 Department of Neonatology and Pediatrics, Noble Hospital and Research Center, Hadapsar, Pune, Maharashtra 411013, India 